\title{
Cryptogenic organizing pneumonia - a rare lung condition
}

\author{
Adriana Grigoras $^{1,2}$, Laura Knieling ${ }^{*}, 1,2$, Diana Iliescu Bulgaru ${ }^{1,2}$ \\ 1 "Grigore T. Popa" University of Medicine and Pharmacy, Iasi, ${ }^{2}$ Institute of Legal Medicine lasi, \\ Romania
}

\begin{abstract}
Cryptogenic organizing pneumonia is characterized by excessive proliferation of granulation tissue within small airways and alveolar ducts associated with chronic inflammation in the surrounding alveoli. Complete resolution occurs in $65-85 \%$ of patients treated with corticosteroid therapy, and recurrence is not uncommon. Within this context, we present the case of a 60 year-old men who died in same day after admission in hospital. Standard microscopy of necroptic specimens revealed edema, vascular congestion, interstitial lung fibrosis, buds of endoalveolar connective tissue and an inflammatory infiltrate in bronchiolar wall. The histopathological examination provides a better understanding of clinical symptoms that lead to dead in this case.

Keywords: cryptogenic organizing pneumonia, BOOP, autopsy
\end{abstract}

\section{Introduction}

Cryptogenic organizing pneumonia is a rare lung pathology described for the first time by Davison in 1983 and 2 years later by Epler under the name idiopathic bronchiolitis obliterans organizing pneumonia (BOOP) [13]. The condition is called "cryptogenic" because the cause is unknown. In this lung disease, the small airways (bronchioles) the tiny air-exchange sacs or alveoli and the walls of small bronchi become inflamed and plugged with connective tissue. Patients present clinical features of an infectious pneumonia (cough, dyspnea and flulike illness) which fails to respond to antibiotic therapy [4, 5]. This type of pneumonia is generally characterized by a favorable prognosis with corticosteroid therapy $[6,7]$.

Received: March 2016; Accepted after review: May 2016; Published: June 2016

*Corresponding author: Laura Knieling, "Grigore T. Popa" University of Medicine and Pharmacy, Iași; Institute of Legal Medicine, Buna Vestire Street, 700455 lasi, Romania.

E-mail: laura knieling@yahoo.com
Cryptogenic organizing pneumonia is discriminated with secondary organizing pneumonia which appears with collagen vascular disease, infection and drug reaction [8-10].

\section{Case report}

We present the case of a 60 year-old man, nonsmoker, who died in less than 24 hours after admission. He had no known history of any respiratory pathology and he was not taking any regular medications. Physical examination revealed tachycardia, tachypnea, cough, fever of $38.3^{\circ} \mathrm{C}$ and normal blood pressure. A chest radiograph showed multiple subpleural areas of consolidation. Necroptic examination has been associated to collection of tissue specimens for microscopy. Paraffinembedding followed by routine hematoxylineosin (HE) and trichrome Masson staining have been performed. The microscopic examination was done using a Leica microscope. 
The necropsy revealed the presence of a cerebral and lung edema, coronary arteries atherosclerosis and subepicardic ischemic myocardial fibrosis, nephrosclerosis, liver steatosis and incipient pancreatic sclerosis.

Routine microscopy confirmed macroscopically lesions. Lung parenchyma showed marked congestion and thickening of the alveolar septa by fibrosis (Figure 1) accompanied by loose plugs of proliferating fibroblasts within the alveolar ducts and airspaces (Figures 2-4).

There were no significant inflammatory changes at the alveolar septa or other chronic granulomatous inflammation in the lung parenchyma.

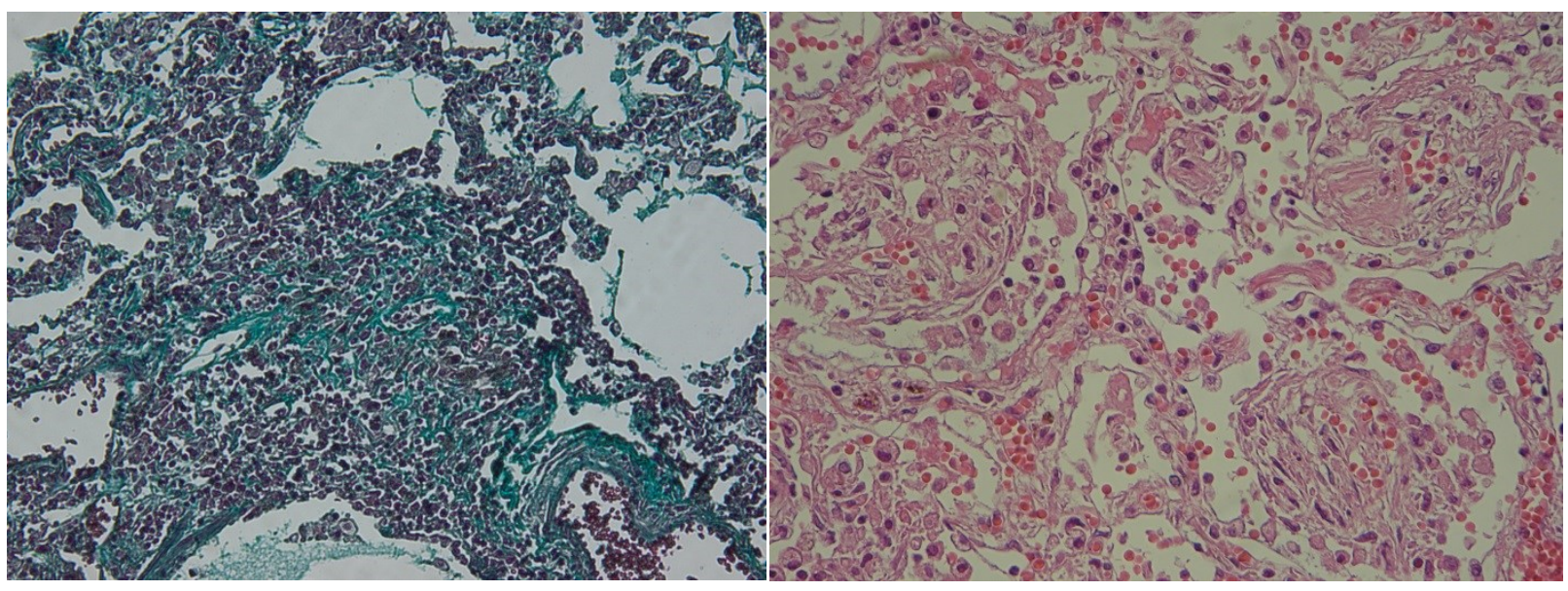

Fig. 1. Fibrous thickening of the alveolar septa (trichrome Masson, x 100)

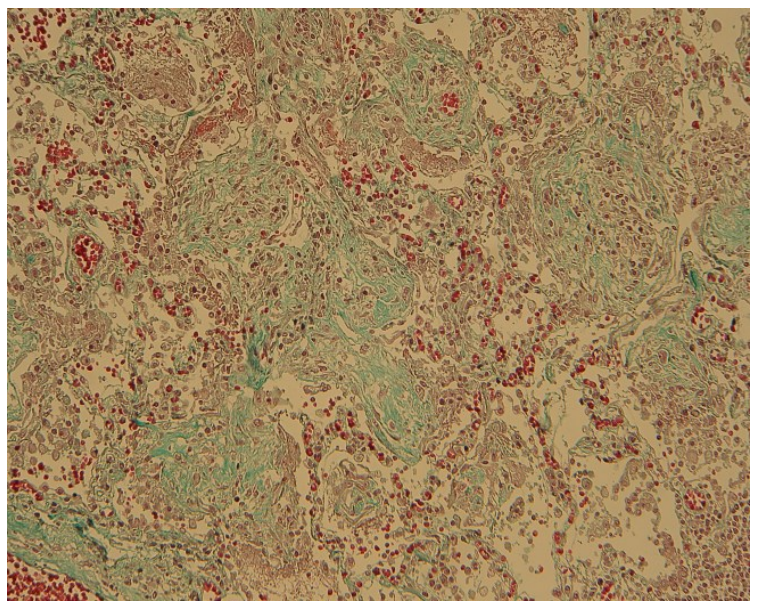

Fig. 3. Multiple fibroblastic plugs within alveoli (trichrome Masson, x 100)

Moreover, varying degrees of mononuclear cells inflammation of the bronchiolar walls involvement have been noticed (Figures 5-6).
Fig. 2. Parietal capillary congestion and fibroblastic plugs in alveolar spaces (HE, x 200)

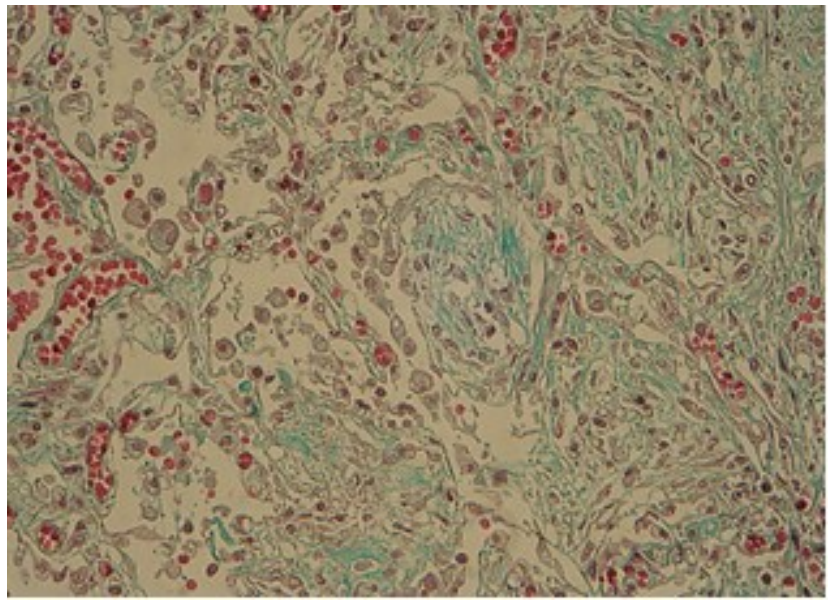

Fig. 4. Detail of Fig. 3 (trichrome Masson, x 200)

Also, microscopic examination revealed the absence of acute inflammatory lesions in the lung parenchyma. 


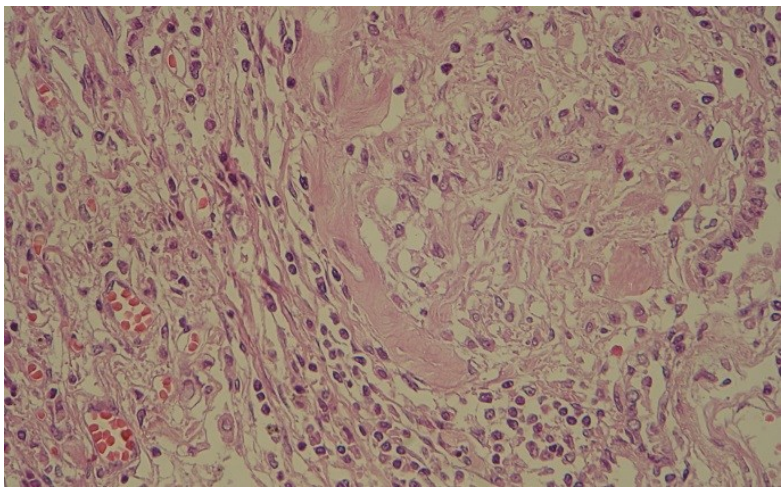

Fig. 5. Chronic inflammation and fibrosis in the bronchiolar wall (HE, x 400)

\section{Discussions}

According to the American Thoracic Society and European Respiratory Society, the idiopathic interstitial pneumonias are classified today into: (1) chronic fibrosing idiopathic interstitial pneumonias (idiopathic pulmonary fibrosis and idiopathic nonspecific interstitial pneumonia); (2) smoking-related idiopathic interstitial pneumonias (respiratory bronchiolitis-associated interstitial lung disease and desquamative interstitial pneumonia); (3) acute or subacute idiopathic interstitial pneumonias (cryptogenic organizing pneumonia and acute interstitial pneumonia) and (4) rare idiopathic interstitial pneumonias (lymphoid interstitial pneumonia and idiopathic pleuroparenchymal fibroelastosis) [11].

The risk of cryptogenic organizing pneumonia is higher for patients with inflammatory diseases like systemic lupus erythematosus and rheumatoid arthritis [12].

The classic presentation of this type of pneumonia is the development of nonspecific systemic (fevers, night sweats, fatigue, weight loss) and respiratory (dyspnea and cough) symptoms in association with multiple bilateral patchy lung opacities, visible on chest radiography [13].

Morphologically, the hallmark of the cryptogenic organizing pneumonia is represented by fibroblastic plugs ("Masson bodies") filling air spaces. These plugs are formed by fibroblasts embedded in a palestaining matrix. Other changes include clusters of foamy macrophages, a few scattered neutrophils, and thickening of the alveolar septa.

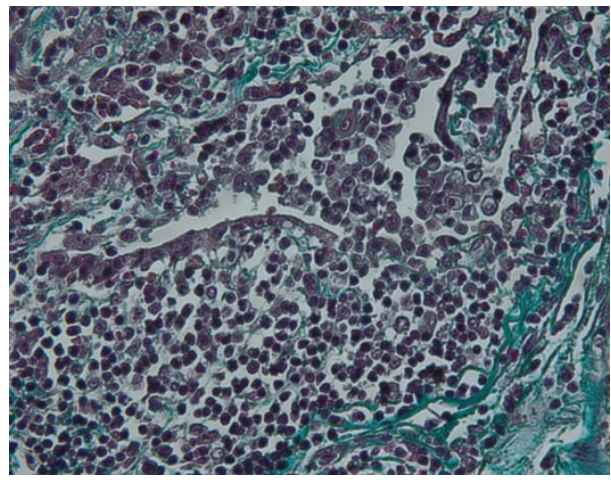

Fig. 6. Chronic inflammation associated with focal fibrosis in the bronchiolar wall (trichrome Masson, $x$ 400)

Organizing pneumonia was initially described by Laennec, as a failure of resolution of acute Pneumococcal pneumonia.

Today, the pathophysiology of cryptogenic organizing pneumonia is a model of a lung fibro-inflammatory disease. In this process, the first step is represented by the acute alveolar epithelial injury with cell necrosis and denudation of the basal laminae. After these epithelial lesions, in the alveolar spaces appear fibrinoid, inflammatory cell clusters rich in coagulation factors and interstitial fibroblasts. These cells undergo phenotypic modulation into myofibroblasts and organize into fibro-inflammatory buds with deposition of a fibrotic connective tissue matrix, but the alveolar architecture is preserved [14].

Organizing pneumonia is called "secondary" when a cause such as an infection, drug toxicity, or a connective tissue disease can be identified.

In our case, the diagnosis based on clinical and paraclinical manifestations was difficult. Only microscopic examination established the cause that led to patient's death.

In Romania, interstitial lung diseases are a group of rare diseases, with difficult diagnosis and management. According to the results of a retrospective study that was recently conducted in "Marius Nasta" Institute of Pulmonology Bucharest, from 178 patients with interstitial lung disease, only 9 cases have been diagnosed with cryptogenic organizing pneumonia [15].

The differential diagnoses for this case included community acquired pneumonia, secondary organizing pneumonia, pulmonary 
embolism with associated infarction or atypical viral or fungal infection [16].

\section{Conclusions}

Although rapidly fatal, cryptogenic organizing pneumonia is rare, respiratory failure leading to death may occur, as in this case. Diagnosis can be improved by a multidisciplinary approach of each patient. Pathological examination is an important step

\section{References}

1. Davison AG, Heard BE, McAllister WA, et al. Cryptogenic organizing pneumonitis. $Q \mathrm{~J}$ Med 1983; 52(207):382-394.

2. Epler GR, Colby TV, McLoud TC, et al. Bronchiolitis obliterans organizing pneumonia. N Engl J Med 1985; 312:152-158.

3. Lebowitz D, Rochat T. Cryptogenic organizing pneumonia. Rev Med Suisse 2013; 9 (407):2164-2169.

4. Baque-Juston $M$, Pellegrin $A$, Leroy $S$, et al. Organizing pneumonia: what is it? A conceptual approach and pictorial review. Diagn Interv Imaging 2014; 95(9):771-777.

5. Zhou H, Gu W, Li C. Post-Infectious Organizing Pneumonia: an Indistinguishable and Easily Misdiagnosed Organizing Pneumonia. Clin Lab 2015; 61(11):1755-1761.

6. Ruth-Sahd LA, White KA. Bronchiolitis obliterans organizing pneumonia. Dimens Crit Care Nurs 2009; 28(5):204-208.

7. Petitpierre N, Beigelman C, Letovanec I, Lazor R. Cryptogenic organizing pneumonia. Rev Mal Respir 2016; S0761-8425(15)01110-9.

8. Vasu TS, Cavallazzi R, Hirani A, et al. Clinical and radiologic distinctions between secondary bronchiolitis obliterans organizing pneumonia and cryptogenic organizing pneumonia. Respir Care 2009; 54(8):1028-3102.

9. Shen W, Li H, Dai J, et al. Analysis for differences in clinical and radiologic findings between patients with cryptogenic organizing pneumonia and connective tissue disorder related organizing pneumonia. Zhonghua Jie He He Hu Xi Za Zhi 2015; 38 (9):669-674. in differentiation between cryptogenic and secondary organizing pneumonia and provides a better understanding of clinical manifestations that led to patient's death.

\section{Conflict of interest}

The authors declare that they have no competing interests.

10. Huo $Z$, Feng $R$, Tian $X$, et al. Clinicopathological findings of focal organizing pneumonia: a retrospective study of 37 cases. Int J Clin Exp Pathol 2015; 8(1):511-516.

11. Sverzellati N, Lynch DA, Hansell DM, et al. American Thoracic Society-European Respiratory Society Classification of the Idiopathic Interstitial Pneumonias: Advances in Knowledge since 2002. Radiographics 2015; 35(7):1849-1871.

12. Sara AG, Hamdan AJ, Hanaa $B$, et al. Bronchiolitis obliterans organizing pneumonia: Pathogenesis, clinical features, imaging and therapy review. Ann Thorac Med 2008; 3 (2):67-75.

13. Nogi $S$, Nakayama $H$, Tajima $Y$, et al. Cryptogenic organizing pneumonia associated with radiation: A report of two cases. Oncol Lett 2014; 7(2):321-324.

14. Cordier JF. Update on cryptogenic organising pneumonia (idiopathic bronchiolitis obliterans organising pneumonia). Swiss Med Wkly 2002; 132(41-42):588-591.

15. Strâmbu I, Belaconi I, Stoicescu I, et al. Interstitial lung diseases: an observational study in patients admitted in "Marius Nasta" Institute of Pulmonology Bucharest, Romania, in 2011. Pneumologia 2013; 62(4):206-211.

16. Alnimer $\mathrm{Y}$, Salah $\mathrm{S}$, Abuqayas $\mathrm{B}$, Alrabi K. Azacitidine-induced cryptogenic organizing pneumonia: a case report and review of the literature. J Med Case Rep 2016; 10:15. 